\title{
Induced circular dichroism of an optically active polyfluorene derivative in phase-separating solutions
}

\author{
Yusuke Sanada and Takahiro Sato
}

When methanol, a nonsolvent, was added to dilute tetrahydrofuran solutions of an optically active polyfluorene derivative, liquid-liquid phase separation occurred, and circular dichroism (CD) was induced at a low temperature. The polymer concentration of the minor separating phase, estimated by light scattering, was very high $\left(\sim 0.4 \mathrm{~g} \mathrm{~cm}^{-3}\right)$. The CD induction occurring in that concentrated phase was temperature sensitive. When the phase-separating solution was quenched from $\mathbf{4 0}$ to $15^{\circ} \mathrm{C}$, the $\mathrm{CD}$ increased according to first-order reaction kinetics, and it was a rather slow process (the rate constant was $2.5 \times 10^{-4} \mathrm{~s}^{-1}$ ). The intermolecular chiral interaction in the concentrated phase may be responsible for the CD induction or non-racemization of this helical polyfluorene derivative in phase-separating solutions. Polymer Journal (2010) 42, 195-200; doi:10.1038/pj.2009.330; published online 23 December 2009

Keywords: $\pi$-conjugated polymer; $C D$; helical polymer; light scattering; phase separation; polyfluorene

\section{INTRODUCTION}

Polyfluorene, a $\pi$-conjugated polymer, has interesting electrical and optical properties and attracts increasing interest as a candidate for organic light-emitting diodes with high quantum yield and high hole mobility. ${ }^{1,2}$ Circular dichroism (CD), optical activity and circular polarized luminescence are additional optical and electro-optical properties that enable polyfluorene to be used in optical devices or sensors. Several researchers ${ }^{3-7}$ were interested in these properties and investigated optically active polyfluorene derivatives bearing chiral side chains.

Molecular modeling on the basis of $a b$ initio molecular orbital calculations indicated that the energetically favorable conformation of the polyfluorene backbone is a $5 / 2$ or $5 / 1$ helix, ${ }^{8,9}$ and this helical conformation was supported by electron and $\mathrm{x}$-ray diffraction from the spin-coated film and fiber of a polyfluorene derivative, ${ }^{8,10}$ as well as from a solution study. ${ }^{6}$ However, dilute solutions of optically active polyfluorene derivatives usually exhibit little CD. ${ }^{6,7}$ This implies that the optically active side chain hardly differentiates between the energies of the right- and left-handed helical conformations of the polyfluorene backbone. On the other hand, strong $\mathrm{CD}$, circular selective reflection and circular polarized luminescence were observed for optically active polyfluorene derivative films, showing that chiral discrimination arises from intermolecular interaction in the condensed phase. ${ }^{3,4,11}$

Some $\pi$ - and $\sigma$-conjugated polymers, for example, polythiophene and polysilylene derivatives, were reported to induce strong CD in dilute solutions by aggregation or phase separation. ${ }^{12-15}$ In these aggregation- and phase-separation-induced CDs, the intermolecular chiral interaction among conjugated polymer chains may have an important role. However, the detailed mechanism of these phenomena has not been elucidated.
Recently, Wu and Sato ${ }^{7}$ reported aggregation-induced CD in dilute tetrahydrofuran (THF) and toluene solutions of two optically active polyfluorene derivatives on cooling to as low as $-80^{\circ} \mathrm{C}$. The observed exciton-coupling signal of $\mathrm{CD}$ arising from the polyfluorene main chain was inverted by changing the solvents (THF and toluene) or through tiny differences in the side-chain chemical structure. Owing to the difficulty in the characterization of polyfluorene aggregates in solution at $-80^{\circ} \mathrm{C}$, the detailed mechanism of the aggregationinduced $\mathrm{CD}$ was not investigated in the previous study.

In this study, we have investigated the solvent condition in which an optically active polyfluorene derivative exhibits $C D$ in a dilute solution near room temperature. $\operatorname{Poly}(2,7-[9,9-b i s((S)$-citronellyl)]fluorene) (PCF, Scheme 1) was chosen as the test polymer, and phase-separation-induced CD was found in dilute THF solutions of this polymer near room temperature by adding a nonsolvent, methanol. To elucidate the detailed mechanism of the $\mathrm{CD}$ induction, we have investigated the kinetics of $\mathrm{CD}$ induced on cooling in the THF-methanol solution, as well as the separated droplet phase, by light scattering measurements. On the basis of those experimental results, we have proposed a model for CD induction in phaseseparating solutions of helical polymers.

\section{EXPERIMENTAL PROCEDURE}

Polymer samples

The dibromofluorene monomer was polymerized in a hot mixture of toluene and $\mathrm{N}, \mathrm{N}$-dimethylformamide using a zero-valent nickel reagent by the Yamamoto coupling reaction. ${ }^{5}$ The polymer was divided into seven fractions by fractional precipitation using toluene as the solvent and methanol as the precipitant. Two fractions, PCF2-1 and PCF2-3, were chosen for the following 


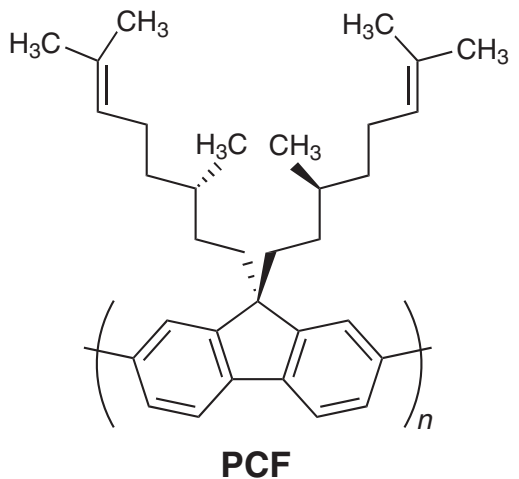

Scheme 1 Repeating unit of PCF.

experiments. The solvents THF and methanol were distilled under calcium hydrate as a desiccant.

\section{Turbidity}

Methanol was added dropwise under stirring to THF solutions of the fraction PCF2-1 with different polymer concentrations, and the turbidity of each solution was observed at room temperature by eye to construct the ternary phase diagram of PCF, THF and methanol. In what follows, the composition of the ternary system is expressed in terms of the volume fraction $\phi_{\mathrm{MeOH}}$ of methanol at the mixing of THF and methanol and the PCF mass concentration $c$ in the total solution.

\section{CD and UV-VIS absorption}

$\mathrm{CD}$ and ultraviolet-visible light (UV-VIS) absorption spectra were measured on phase-separating solutions of fraction PCF2- 1 with $\phi_{\mathrm{MeOH}}=0.5$ and different $c$ values using a JASCO J-720WO spectropolarimeter (JASCO, Tokyo, Japan) at 40 and $15^{\circ} \mathrm{C}$. A quartz cell with a $10-\mathrm{cm}$ optical pass length and a thermostat jacket was used for the measurements.

\section{Light scattering}

Static light scattering measurements were carried out on THF and methanoladded THF solutions of samples PCF2-1 and PCF2-3 using a Fica 50 light scattering photometer with vertically polarized incident light of $546 \mathrm{~nm}$ and without an analyzer. The light scattering systems were calibrated using toluene as the reference material.

For THF solutions of the two PCF samples, the Rayleigh ratio $R_{\theta}$ excess over that of the solvent obtained was analyzed by the conventional Berry plot to determine the weight-average molar mass $M_{\mathrm{w}}$, the second virial coefficient $A_{2}$, and the $z$-average square radius of gyration $\left\langle S^{2}\right\rangle$ using the equations ${ }^{16}$

$$
\begin{aligned}
\lim _{\theta \rightarrow 0}\left(\frac{K c}{R_{\theta}}\right)^{1 / 2} & =\frac{1}{M_{\mathrm{w}}^{1 / 2}}+A_{2} M_{\mathrm{w}}^{1 / 2} c+\cdots, \lim _{c \rightarrow 0}\left(\frac{K c}{R_{\theta}}\right)^{1 / 2} \\
& =\frac{1}{M_{\mathrm{w}}^{1 / 2}}\left(1+\frac{1}{6}\left\langle S^{2}\right\rangle k^{2}+\cdots\right)
\end{aligned}
$$

where $K$ is the optical constant and $k$ is the magnitude of the scattering vector. The results of $M_{\mathrm{w}}, A_{2}$ and $\left\langle S^{2}\right\rangle^{1 / 2}$ for samples PCF2-1 and PCF2-3 in THF are listed in Table 1.

Light scattering from the polymer in a mixed solvent is affected by preferential adsorption. Owing to this effect, equation (1) should be replaced by the following equation, ${ }^{17}$

$$
\begin{aligned}
& \lim _{c \rightarrow 0}\left(\frac{K c}{R_{\theta}}\right)^{1 / 2}\left[1+\left(\frac{\partial c_{1}}{\partial c}\right)_{\mu_{1}} \frac{\left(\partial \tilde{n} / \partial c_{1}\right)_{c}}{(\partial \tilde{n} / \partial c)_{c_{1}}}\right] \equiv \lim _{c \rightarrow 0}\left(\frac{K^{*} c}{R_{\theta}}\right)^{1 / 2} \\
& =\frac{1}{M_{\mathrm{w}}^{1 / 2}}\left(1+\frac{1}{6}\left\langle S^{2}\right\rangle k^{2}+\cdots\right)
\end{aligned}
$$

where $\left(\partial c_{1} / \partial c\right)_{\mu_{1}}$ is the increment of the mass concentration $c_{1}$ of the secondary solvent (methanol in our system) with increasing $c$ at a constant
Table 1 Molecular characteristics of the PCF samples used

\begin{tabular}{cccc}
\hline Sample & $M_{\mathrm{w}} / 10^{5}$ & $A_{2} / 10^{-3} \mathrm{~cm}^{3} \mathrm{molg}^{-2}$ & $\left\langle S^{2}\right\rangle^{1 / 2} \mathrm{~nm}^{-1}$ \\
\hline PCF2-1 & 1.21 & 1.32 & 35.3 \\
PCF2-3 & 1.29 & 1.38 & 31.6
\end{tabular}

Abbreviation: PCF, poly(2,7-[9,9-bis((S)-citronellyl)]fluorene).

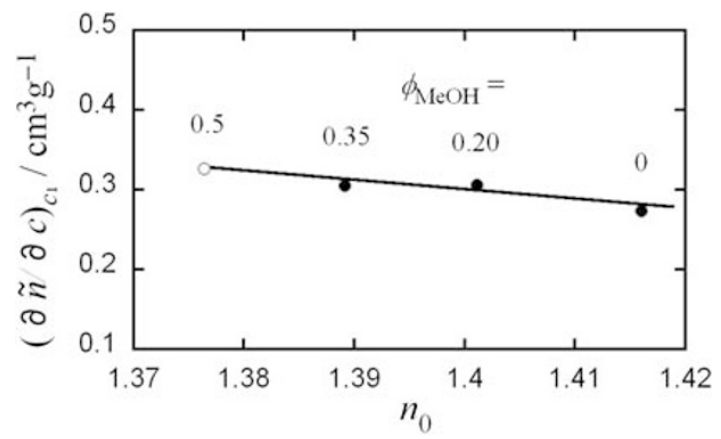

Figure 1 Specific refractive index increments of PCF in THF and THFmethanol mixtures at $30^{\circ} \mathrm{C}$.

solvent chemical potential, and $(\partial \tilde{n} / \partial c)_{c_{1}}$ and $\left(\partial \tilde{n} / \partial c_{1}\right)_{c}$ are the specific refractive index increments of the polymer and the secondary solvent components, respectively.

The specific refractive index increment $(\partial \tilde{n} / \partial c)_{c_{1}}$ at constant solvent composition, included in $K$, was measured at $30^{\circ} \mathrm{C}$ using a differential refractometer of the modified Schulz-Cantow type. Figure 1 shows the results of $(\partial \tilde{n} / \partial c)_{c_{1}}$ at $\phi_{\mathrm{MeOH}}\left(c_{1}\right)=0 \quad\left(0 \mathrm{~g} \mathrm{~cm}^{-3}\right), 0.2\left(0.21 \mathrm{~g} \mathrm{~cm}^{-3}\right)$ and $0.35\left(0.38 \mathrm{~g} \mathrm{~cm}^{-3}\right)$, represented by filled circles. The value of $(\partial \tilde{n} / \partial c)_{c_{1}}$ at $\phi_{\mathrm{MeOH}}\left(c_{1}\right)=0.5$ $\left(0.57 \mathrm{~g} \mathrm{~cm}^{-3}\right)$ was obtained by extrapolation (unfilled circle in Figure 1).

\section{RESULTS AND DISCUSSION}

Phase diagram

Figure 2 illustrates the phase diagram of the ternary system of sample PCF2-1, THF and methanol at room temperature. Here, $c$ is the polymer mass concentration, $\phi_{\mathrm{MeOH}}$ is the volume fraction of methanol at the mixing of THF and methanol, and the unfilled and filled circles indicate single-phase and biphasic states, respectively. This is a typical ternary phase diagram of a polymer, solvent and nonsolvent. ${ }^{18}$ CD and UV-VIS absorption, as well as light scattering measurements, mentioned below, were made in the two-phase region mostly at fixed $\phi_{\mathrm{MeOH}}=0.5$.

\section{CD and UV-VIS absorption}

Figure 3 shows CD and UV-VIS absorption spectra of sample PCF2-1 in the THF-methanol mixture of $\phi_{\mathrm{MeOH}}=0.5\left(c=6.0 \times 10^{-7} \mathrm{~g} \mathrm{~cm}^{-3}\right)$. Although the solution is $\mathrm{CD}$ inactive at $40^{\circ} \mathrm{C}$, a bisigned signal grows on cooling of the solution to $15^{\circ} \mathrm{C}$. Although the peak height of the UV-VIS absorption decreases with the cooling time, that of the CD spectrum increases, except at $300 \mathrm{~min}$. The diminishment of the absorption spectrum may arise from the increase of scattering from the solution; the scattered light cannot contribute to the absorption. (Although not shown, the baseline of the original UV-VIS absorption curve was considerably dependent on the wavelength $\lambda$ according to Rayleigh's $\lambda^{-4}$ law $^{16}$ and increased with time at $15^{\circ} \mathrm{C}$. This baseline was subtracted from the original absorption curve to obtain $A$, shown in Figure 3). Similar CD and UV-VIS absorption spectra were obtained at different $c$ values $\left(=2.0 \times 10^{-7}\right.$ and $4.0 \times 10^{-7}$ $\mathrm{g} \mathrm{cm}^{-3}$ ). These CD inductions were almost reversible, that is, when 


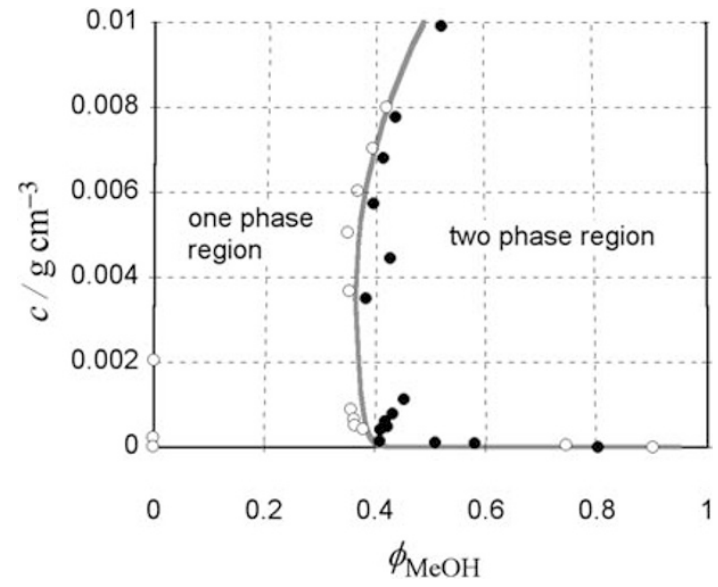

Figure 2 Phase diagram of the ternary system PCF, THF and methanol at room temperature; unfilled circles, one-phase region; filled circles, twophase region. A full color version of this figure is available at the Polymer Journal online.
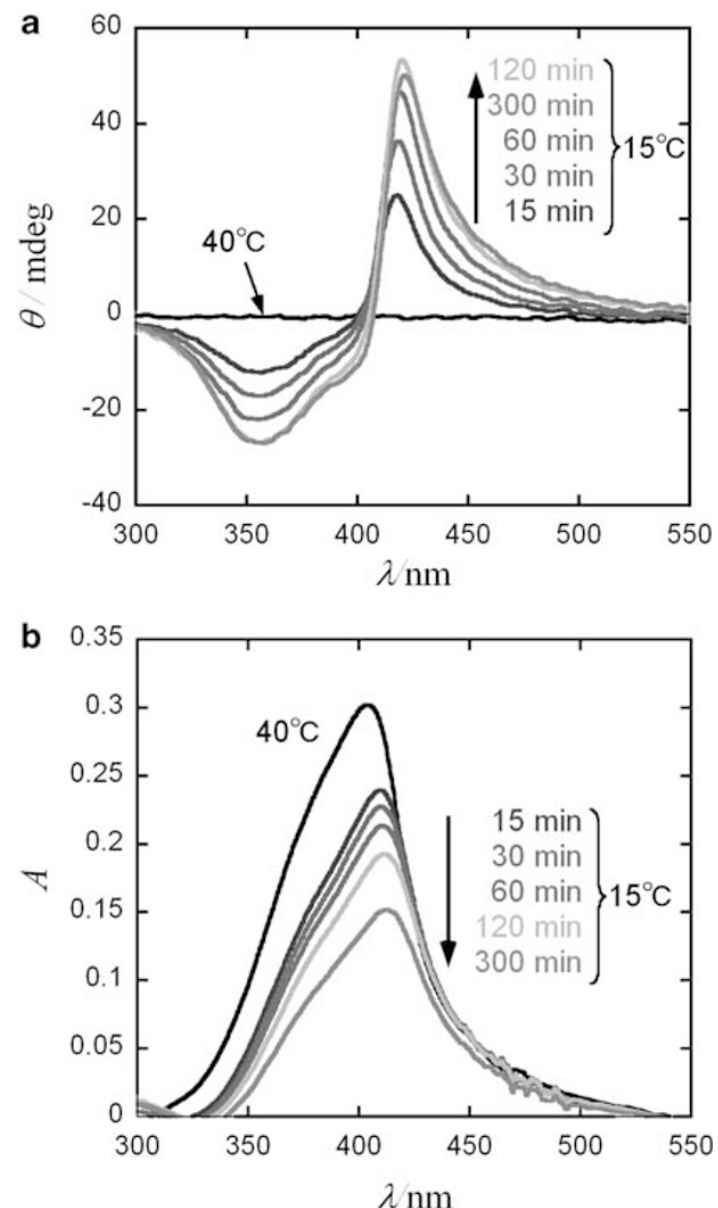

Figure 3 (a) CD and (b) UV-VIS absorption spectra for a phase-separating solution of sample PCF2-1 $\left(\phi_{\mathrm{MeOH}}=0.5, c=6.0 \times 10^{-7} \mathrm{~g} \mathrm{~cm}^{-3}\right)$ at $40^{\circ} \mathrm{C}$, quenched to $15^{\circ} \mathrm{C}$. A full color version of this figure is available at the Polymer Journal online.

the quenched solutions were heated to $40{ }^{\circ} \mathrm{C}$, the induced $\mathrm{CD}$ vanished again.

In Figure 3, the UV-VIS absorption peak seems to show a slight red shift. If this red shift reflects the transformation to the $\beta$-phase of polyfluorene chains, ${ }^{19}$ the peak should return to the original position on heating to $40^{\circ} \mathrm{C}$. However, the red shift was not reversible. The UV-VIS absorption experiment for the phase-separating solutions is affected by the wavelength-dependent scattering, and the baseline subtraction may not be enough to perfectly correct this scattering effect. Owing to this experimental uncertainty, we do not argue the origin of the small peak shift here.

The induced CD must arise from the separating concentrated phase, and the molar $\mathrm{CD} \Delta \varepsilon_{\mathrm{c}}$ of the concentrated phase is calculated from the observed ellipticity $\theta$ by

$$
\Delta \varepsilon_{\mathrm{c}}=\frac{4 \pi \log e}{180} \frac{\theta}{\left(1000 c_{\mathrm{c}} / M_{0}\right) \Phi l}
$$

where $c_{\mathrm{c}}$ and $\Phi$ are the mass concentration and the volume fraction (in the total solution) of the concentrated phase, respectively, $M_{0}$ is the molar mass of the PCF repeating unit and $l$ is the path length; $\Phi l$ represents the average path length of the concentrated phase. On the other hand, the average molar extinction coefficient $\varepsilon_{\mathrm{c}}$ of the concentrated phase is calculated from the observed absorbance $A$ using

$$
\varepsilon_{\mathrm{c}}=\frac{A\left(c-c_{\mathrm{d}}\right) / c}{\left(1000 c_{\mathrm{c}} / M_{0}\right) \Phi l}
$$

where $c_{\mathrm{d}}$ is the mass concentration of the coexisting dilute phase. Thus, the Kuhn dissymmetry factor of the concentrated phase may be proportional to the following quantity: ${ }^{20}$

$$
g_{\mathrm{c}} \equiv \Delta \varepsilon_{\mathrm{c}, \mathrm{m}} / \varepsilon_{\mathrm{c}, \mathrm{m}}=\frac{4 \pi \log e}{180} \frac{\theta_{m}}{A_{\mathrm{m}}\left(c-c_{\mathrm{d}}\right) / c} .
$$

Here, $\theta_{\mathrm{m}}$ and $A_{\mathrm{m}}$ are the peak heights of ellipticity and absorbance, respectively. The value of $c_{\mathrm{d}}$ was estimated by light scattering (cf. Figure 6).

Figure 4 displays the time evolution of $g_{\mathrm{c}}$ of the solutions shown in Figure 3 after quenching from 40 to $15^{\circ} \mathrm{C}$. The data points can be fitted to single-exponential functions (solid curves in the figure), although the initial and final $g_{c}$ values are slightly different at each polymer concentration $c$. Therefore, the CD induction obeys first-order reaction kinetics (cf. the discussion at the end of this section). From the fitting curves, the reaction rate constant was estimated to be $2.5 \times 10^{-4} \mathrm{~s}^{-1}$. Thus, the CD induction is a rather slow molecular event.

\section{Light scattering}

Figure 5 compares $\left(K c / R_{\theta}\right)^{1 / 2}$ at $\theta \rightarrow 0$ for sample PCF2-3 in THF and a THF-methanol mixture with $\phi_{\mathrm{MeOH}}=0.2$. The disagreement of the intercepts comes from preferential adsorption, and using equation (2) we can estimate the degree of preferential adsorption $\left(\partial_{c 1} / \partial_{c}\right)_{\mu_{1}}$ to be -0.025 . [ At $\phi_{\mathrm{MeOH}}=0.2,\left(\partial \tilde{n} / \partial c_{1}\right)_{c=0}=-0.089 \mathrm{~cm}^{3} \mathrm{~g}^{-1}$ ]. Neglecting the solvent composition dependence of $\left(\partial c_{1} / \partial c\right)_{\mu_{1}}$, we may calculate the optical constant $K^{*}$ in equation (2) at $\phi_{\mathrm{MeOH}}=0.5$ using $\left(\partial \tilde{n} / \partial c_{1}\right)_{c=0}=-0.11 \mathrm{~cm}^{3} \mathrm{~g}^{-1}$ and $\left(\partial \tilde{n} / \partial c_{1}\right)_{c_{1}}=0.326 \mathrm{~cm}^{3} \mathrm{~g}^{-1}$ (cf. Figure 1).

At $\phi_{\mathrm{MeOH}}=0.5, R_{\theta}$ is high enough, even for very dilute solutions, because droplets of the minor concentrated phase possess very strong scattering power. In Figure $6,\left(R_{\theta} / K^{\star}\right)^{1 / 2}$ at $\theta \rightarrow 0$ for sample PCF2-1 at $\phi_{\mathrm{MeOH}}=0.5$ and $40^{\circ} \mathrm{C}$ is plotted against $c$. From this plot, $R_{\theta}$ seems to vanish at $\sim 3 \times 10^{-8} \mathrm{~g} \mathrm{~cm}^{-3}$. This critical concentration can be regarded as the polymer concentration $c_{\mathrm{d}}$ of the coexisting dilute phase. In what follows, we are interested in droplets of the minor concentrated phase in the solution, which is responsible for the CD induction. The mass concentration of the concentrated phase droplets in the solution is given by $c-c_{\mathrm{d}}$. Strictly speaking, $c_{\mathrm{d}}$ slightly depends on 


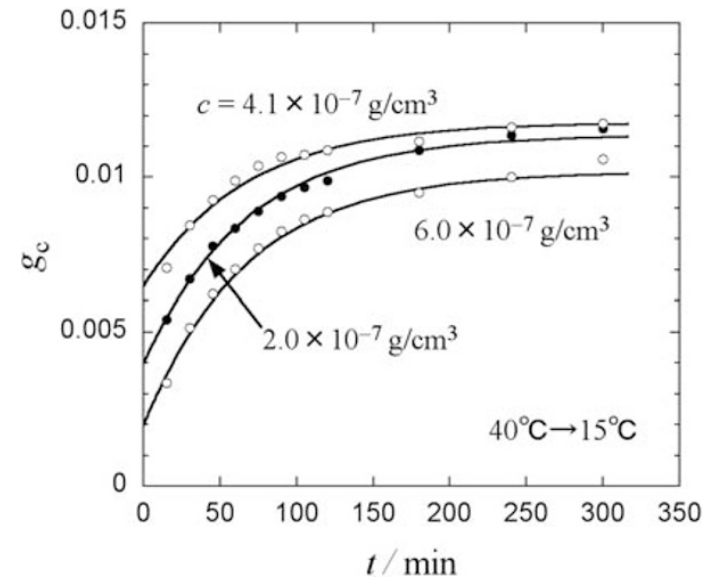

Figure 4 Time evolution of the Kuhn dissymmetry factor for the concentrated phase in the phase-separating solution shown in Figure 3 after quenching from 40 to $15{ }^{\circ} \mathrm{C}$; solid curves, single-exponential fitting results (cf. equation (15)).

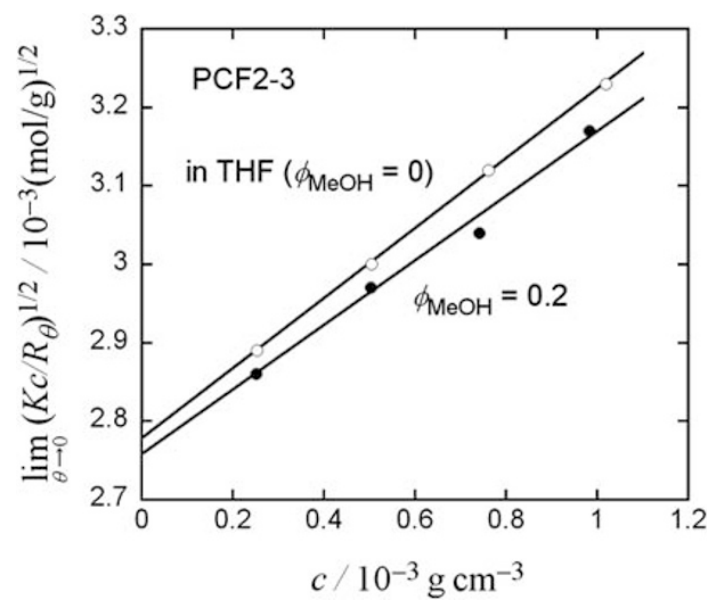

Figure 5 Concentration dependences of $\left(K c / R_{\theta}\right)^{1 / 2}$ at $\theta \rightarrow 0$ for sample PCF2-3 in THF and a THF-methanol mixture with $\phi_{\mathrm{MeOH}}=0.2$.

$c$, but within the dilute $c$ range we examined $c_{\mathrm{d}}$ may be approximated to be $3 \times 10^{-8} \mathrm{~g} \mathrm{~cm}^{-3}$, as determined in Figure 6, irrespective of $c$.

Figure 7 compares $\left[K^{\star}\left(c-c_{\mathrm{d}}\right) / R_{\theta}\right]^{1 / 2}$ for the concentrated droplet phase in a solution of sample PCF2-1 $\left(\phi_{\mathrm{MeOH}}=0.5, c=6.0 \times 10^{-7} \mathrm{~g} \mathrm{~cm}^{-3}\right)$ at $40^{\circ} \mathrm{C}$ and on cooling to $15^{\circ} \mathrm{C}$. The scattering intensity and thus the size and amount of the droplet phase in the solution change little on cooling. Similar temperature-insensitive light scattering results were also obtained for solutions of sample PCF2-1 with $\phi_{\mathrm{MeOH}}=0.5$ and different $c$ values $\left(2.0 \times 10^{-7}\right.$ and $\left.4.1 \times 10^{-7} \mathrm{~g} \mathrm{~cm}^{-3}\right)$. This is in sharp contrast to the CD induction shown in Figure 3, indicating that phase separation may be a necessary condition, but not a sufficient one, for CD induction.

Let us assume that the droplets of the concentrated phase are polydisperse spherical particles obeying a log-normal distribution. The log-normal distribution ${ }^{21,22}$ is expressed in terms of the weight fraction $w(M)$ of the molar mass $M$ given by

$$
w(M)=\frac{1}{\sqrt{\pi} \beta M} \exp \left\{-\left[\beta^{-1} \operatorname{In}\left(M / M^{o}\right)\right]^{2}\right\}
$$

where

$$
M^{\circ}=\sqrt{M_{\mathrm{w}} M_{\mathrm{n}}}, \beta \equiv \sqrt{2 \ln \left(M_{\mathrm{w}} / M_{\mathrm{n}}\right)}
$$

with weight- and number-average molar masses $M_{\mathrm{w}}$ and $M_{\mathrm{n}}$. The particle scattering function $P(k)$ of the sphere with a molar mass $M$ is

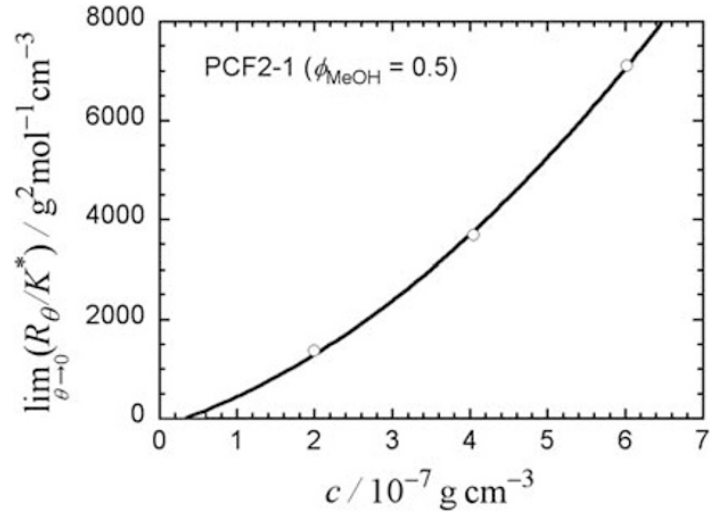

Figure 6 Concentration dependence of $\left(R_{\theta} / K^{*}\right)^{1 / 2}$ at $\theta \rightarrow 0$ for sample PCF2-1 at $\phi_{\mathrm{MeOH}}=0.5$ and $40^{\circ} \mathrm{C}$.

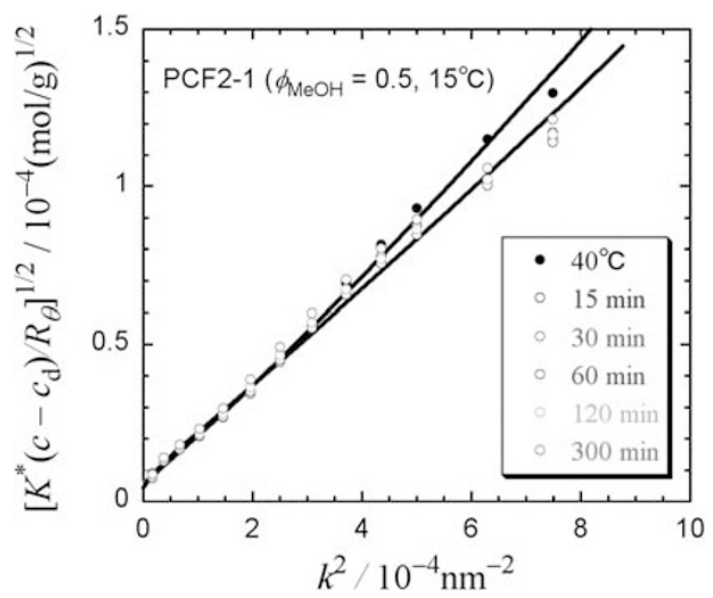

Figure 7 Angular dependences of $\left[K^{\star}\left(c-c_{\mathrm{d}}\right) / R_{\theta}\right]^{1 / 2}$ for the concentrated droplet phase in a solution of sample PCF2- $1 \quad\left(\phi_{\mathrm{MeOH}}=0.5\right.$, $c=6.0 \times 10^{-7} \mathrm{~g} \mathrm{~cm}^{-3}$ ) at $40^{\circ} \mathrm{C}$ and on cooling to $15^{\circ} \mathrm{C}$; solid curves, calculated by equations (6-10) with the fitting parameters listed in Table 2. A full color version of this figure is available at the Polymer Journal online.

given by ${ }^{23}$

$$
P(k)=\frac{9}{(k R)^{6}}[\sin (k R)-k R \cos (k R)]^{2}
$$

where $R$ is the radius of the sphere related to $M$ by

$$
\frac{4 \pi}{3} R^{3} N_{\mathrm{A}} c_{\mathrm{c}}=M
$$

with Avogadro's constant $N_{\mathrm{A}}$ and mass concentration $c_{\mathfrak{c}}$ of the concentrated phase.

Polymer concentrations of the solutions investigated are so dilute $\left(<10^{-6} \mathrm{~g} \mathrm{~cm}^{-3}\right)$ that the interparticle interference effect may be neglected in the scattering intensity. In such a case, $K^{\star}\left(c-c_{\mathrm{d}}\right) / R_{\theta}$ can be calculated by ${ }^{16,24}$

$$
\frac{K^{*}\left(c-c_{\mathrm{d}}\right)}{R_{\theta}}=\frac{1}{M_{\mathrm{w}} P_{\mathrm{z}}(k)}=\left[\int_{0}^{\infty} M P(k) w(M) d M\right]^{-1}
$$

Figure 8 shows fitting results of $K^{\star}\left(c-c_{\mathrm{d}}\right) / R_{\theta}$ for phase-separating solutions of sample PCF2- 1 with $\phi_{\mathrm{MeOH}}=0.5$ and three different $c$ values at $40{ }^{\circ} \mathrm{C}$. The solid curves, drawn using equations (6-10) with the fitting parameters listed in Table 2, almost fit to the experimental data points. The weight-average molar mass $M_{\mathrm{w}}$ of the concentrated droplet phase is of the order of $10^{10}$, indicating that each droplet 


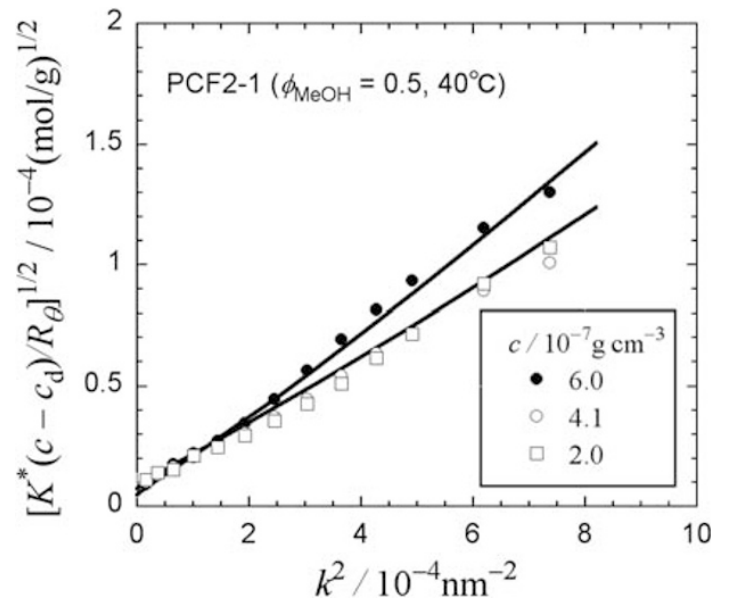

Figure 8 Angular dependences of $\left[K^{*}\left(c-c_{d}\right) / R_{\theta}\right]^{1 / 2}$ for concentrated droplet phases in solutions of sample PCF2- 1 with $\phi_{\mathrm{MeOH}}=0.5$ and three different $c$ values at $40^{\circ} \mathrm{C}$; solid curves, calculated by equations (6-10) with the fitting parameters listed in Table 2 .

Table 2 Fitting parameters characterizing the concentrated droplet phase in solutions of sample PCF2-1 with $\phi_{\mathrm{MeOH}}=0.5$

\begin{tabular}{lcccc}
\hline$c / 10^{-7} \mathrm{gcm}^{-3}$ & Temperature $\left({ }^{\circ} \mathrm{C}\right)$ & $M_{\mathrm{w}} / 10^{10}$ & $M_{\mathrm{w}} / M_{\mathrm{n}}$ & $c_{\mathrm{c}} / \mathrm{gcm}^{-3}$ \\
\hline $2.0,4.1$ & 40 & 2.0 & 20 & 0.43 \\
6.0 & 40 & 5.0 & 20 & 0.42 \\
6.0 & 15 & 5.0 & 50 & 0.42 \\
\hline
\end{tabular}

Abbreviation: PCF, poly(2,7-[9,9-bis((S)-citronellyl)]fluorene).

consists of $\sim 10^{5}$ PCF chains. The molar mass distribution of the droplet phase is wide, ranging from $10^{7}$ to $10^{12}$. The concentration of the concentrated droplet phase is $\sim 0.4 \mathrm{~g} \mathrm{~cm}^{-3}$, and we can expect a strong intermolecular interaction among PCF chains in the concentrated phase. The solid curves in Figure 7 are also theoretical curves calculated by equations (6-10) with the fitting parameters listed in Table 2. The fitting parameters indicate that the molar mass distribution of the droplet phase becomes slightly wider and the smaller droplet phase increases on cooling.

It was verified that the anisotropic light-scattering intensity is almost zero for the phase-separating solution of PCF with $c \sim 10^{-6} \mathrm{~g} \mathrm{~cm}^{-3}$ and $\phi_{\mathrm{MeOH}}=0.5$. This indicates that the concentrated phase may not be the liquid-crystalline phase.

The concentration $c_{\mathrm{c}}$ is much higher than the overlap concentration of sample PCF2-1 ( 10 ${ }^{-3} \mathrm{~g} \mathrm{~cm}^{-3}$; cf. Table 1$)$, and the PCF chains are highly entangled with each other in the concentrated phase. Therefore, the helical sense conversion in PCF chains is rather difficult, which may be responsible for the slow $\mathrm{CD}$ induction shown in Figure 4.

\section{Intermolecular chiral interaction and CD induction}

According to the molecular orbital calculation and molecular modeling, ${ }^{8,9}$ the polyfluorene chain favorably takes a $5 / 2$ or $5 / 1$ helical conformation. However, PCF did not exhibit CD in a dilute THF solution near room temperature. ${ }^{6,7}$ This indicates that PCF chains take right- and left-handed helical conformations with equal probability. On the other hand, in phase-separating THF solutions of PCF by adding methanol, the right- and left-handed helical conformations are discriminated to induce CD because of polyfluorene main-chain absorption. This chiral discrimination may come from some chiral interaction among PCF chains in the separating concentrated droplet phase.
Applying McLachlan's general theory ${ }^{25-28}$ of dispersion interaction, Osipov $^{29-31}$ formulated the chiral attractive interaction potential $w^{*}$ between two chiral objects as follows:

$$
w^{*}=-\frac{J^{*}}{p^{7}}\left[\left(\mathbf{a} \cdot \mathbf{a}^{\prime}\right)-6\left(\mathbf{a} \cdot \frac{\mathbf{p}}{p}\right)\left(\mathbf{a}^{\prime} \cdot \frac{\mathbf{p}}{p}\right)\right]\left(\mathbf{a} \times \mathbf{a}^{\prime} \cdot \frac{\mathbf{p}}{p}\right)
$$

where $\mathbf{a}$ and $\mathbf{a}^{\prime}$ are unit vectors parallel to the principal axes of the two objects, $\mathbf{p}$ is the distance vector between the centers of mass of the two objects, and $J^{\star}$ is the interaction strength calculated by

$$
J^{*} \approx \frac{6 \hbar v^{2}}{16 \pi^{2}} \int_{0}^{\infty} \mathrm{d} \omega \frac{\left[\varepsilon_{\mathrm{m}}^{2}+\varepsilon_{\mathrm{m}}\left(\varepsilon_{\|}-3 \varepsilon_{\perp}\right)+\varepsilon_{\|} \varepsilon_{\perp}\right]\left[\left(2 g_{\|}-g_{\perp}\right) \varepsilon_{\mathrm{m}}-g_{\perp}\right]}{\varepsilon_{\mathrm{m}}\left(\varepsilon_{\mathrm{m}}+\varepsilon_{\perp}\right)^{3}}
$$

Here, $\hbar$ is the Dirac constant, $v$ is the segment volume, $\omega$ is the angular frequency, $\varepsilon_{\mathrm{m}}, \varepsilon_{\|}$and $\varepsilon_{\perp}$ are the solvent permittivity and the longitudinal and transverse components of the segment permittivity, respectively, and $g_{\|}$and $g_{\perp}$ are the longitudinal and transverse components of the segment gyration tensor, respectively. $w^{*}$ is a short-range interaction proportional to $p^{-7}$. Equation (12) may govern the solvent dependence of the induced $\mathrm{CD}{ }^{7}$

The right- or left-handed helical conformation of the PCF chain provides the chirality. Therefore, helical segments of the PCF chain can be regarded as chiral objects, and the chiral interaction $w^{*}$ given by equation (11) is expected among the segments. Although $w^{*}$ should be zero if the segment orientation is perfectly isotropic, we may expect non-zero $w^{*}$ because of the local anisotropic orientation of the segments due to the anisotropic interaction at close approach.

From symmetry, $w^{\star}$ between right-handed helical segments must be of the same magnitude as, and opposite in sign to, that between lefthanded helical segments; also, $w^{*}=0$ between right- and left-handed helical segments. Thus, in the solution of PCF in which the fraction of the right-handed helical segment is $f_{\mathrm{B}}$ the average $w^{*}$ should be proportional to $2 f_{\mathrm{P}}-1$ of the solution.

Including the entropic term, the difference $\Delta G_{\mathrm{h}}$ in the free energy between right- and left-handed helical segments may be written in the form

$$
\Delta G_{\mathrm{h}}=\kappa\left(2 f_{\mathrm{P}}-1\right)
$$

where $\kappa$ is the proportional constant, and its sign is determined by the intrinsic chirality of the PCF chain, that is, its chiral side chain. Lifson et al. ${ }^{32}$ calculated $f_{\mathrm{P}}$ on the basis of the one-dimensional Ising model. Their theory includes three fitting parameters, $\Delta G_{\mathrm{h}}$, the free energy $\Delta G_{\mathrm{r}}$ of the helix reversal and the number of segments $N_{0}$ per chain. Now, we choose the repeating unit of PCF as the segment; $N_{0}=280$ for sample PCF2-1. Although we have no information about $\Delta G_{\mathrm{r}}$ for the PCF chain, here we choose a value of $10 \mathrm{~kJ} \mathrm{~mol}^{-1}$ for $\Delta G_{\mathrm{r}}$. This is the typical $\Delta G_{\mathrm{r}}$ value for helical polymers such as polyacetylene or polyisocyanate derivatives. ${ }^{20,33}$ Figure 9 displays the temperature dependence of $2 f_{\mathrm{P}}-1$ for three different $\kappa$-values on the basis of the Ising model. It can be seen that $2 f_{\mathrm{P}}-1$ sharply increases with decreasing temperature below some critical value. The strong temperature dependence of the induced CD in Figure 3 seems to be consistent with this theoretical result.

When the helical sense conversion of the PCF segment occurs through the segment-segment interaction, there are four elementary reaction processes:

$$
\begin{array}{ll}
\mathrm{M}+\mathrm{P} \stackrel{k_{\mathrm{p}, \mathrm{P}}}{\longrightarrow} 2 \mathrm{P} & \mathrm{M}+\mathrm{M} \stackrel{k_{\mathrm{P}, \mathrm{M}}}{\longrightarrow} \mathrm{P}+\mathrm{M} \\
\mathrm{P}+\mathrm{P} \stackrel{k_{\mathrm{M}, \mathrm{P}}}{\longrightarrow} \mathrm{M}+\mathrm{P} & \mathrm{P}+\mathrm{M} \stackrel{k_{\mathrm{M}, \mathrm{M}}}{\longrightarrow} 2 \mathrm{M}
\end{array}
$$

where $\mathrm{P}$ and $\mathrm{M}$ represent the right- and left-handed helical states of each segment, and $k_{\mathrm{P}, \mathrm{B}} k_{\mathrm{P}, \mathrm{M}}, k_{\mathrm{M}, \mathrm{P}}$ and $k_{\mathrm{M}, \mathrm{M}}$ are rate constants of 


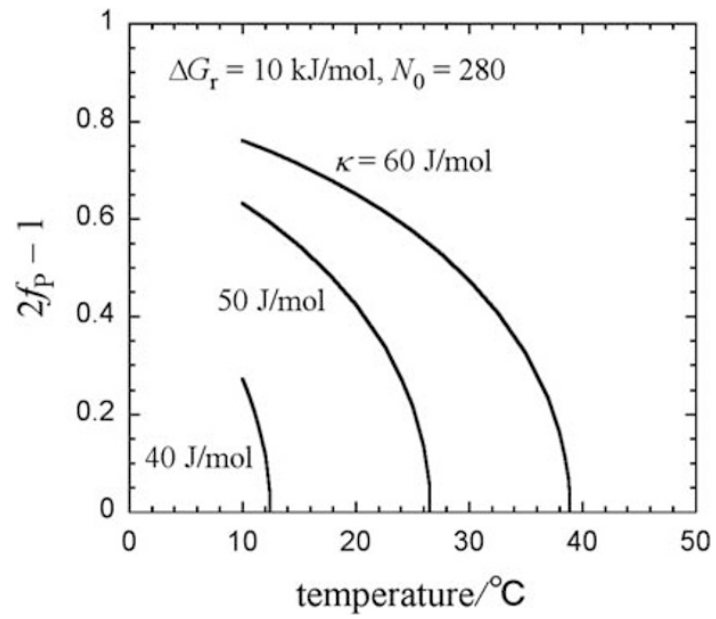

Figure 9 Temperature dependence of $2 f_{\mathrm{p}}-1$ calculated for three different $\kappa$-values on the basis of the Ising model for helical polymers 32 with $N_{0}=280, \Delta G_{\mathrm{r}}=10 \mathrm{~kJ} \mathrm{~mol}^{-1}$, and equation (13).

the elementary reactions. If those elementary reaction rates are determined only by the energies of the initial and final states, we have the relations $k_{\mathrm{P}, \mathrm{P}}=k_{\mathrm{P}, \mathrm{M}}\left(\equiv k_{\mathrm{P}}\right)$ and $k_{\mathrm{M}, \mathrm{P}}=k_{\mathrm{M}, \mathrm{M}}\left(\equiv k_{\mathrm{M}}\right)$ from the above symmetry argument on $w^{*}$. Using these relations, we finally obtain the following kinetic equation of the first-order reaction

$$
2 f_{\mathrm{P}}-1=\frac{k_{\mathrm{P}}-k_{\mathrm{M}}}{k_{\mathrm{P}}+k_{\mathrm{M}}}\left\{1-\exp \left[-\left(k_{\mathrm{P}}+k_{\mathrm{M}}\right) t\right]\right\}
$$

This is consistent with the time evolution of $g_{c}$ shown in Figure 3.

\section{CONCLUSION}

We have investigated the induced CD in phase-separating solutions of an optically active polyfluorene derivative (PCF). In the phaseseparating solutions, the polymer concentration of the minor concentrated phase was very high $\left(\sim 0.4 \mathrm{~g} \mathrm{~cm}^{-3}\right)$. The CD induction occurring in that concentrated phase was temperature sensitive and obeyed first-order reaction kinetics in the quenched solution. This was a rather slow process (the rate constant was $2.5 \times 10^{-4} \mathrm{~s}^{-1}$ ).

The above experimental results can be explained by the following molecular mechanism. In the separating concentrated phase, each PCF segment feels strong chiral interactions from surrounding PCF segments. These interactions may bring about a helical sense conversion, followed by the non-racemization of PCF chains that induces CD. The helical sense conversion by the segment-segment interaction originates from the four elementary reaction processes given by equation (14), which provide first-order reaction kinetics under a certain condition. The temperature sensitivity of the CD induction can be explained on the basis of the Ising model for helical polymers. The slow process of $\mathrm{CD}$ induction may be due to high entanglements among PCF chains in the concentrated phase.

\section{ACKNOWLEDGEMENTS}

This work was partly supported by a Grant-in-Aid for Scientific Research on Priority Area 'Soft Matter Physics.' One of the authors (Y Sanada) expresses his special thanks for the global center of excellence program, 'Global Education and Research Center for Bio-Environmental Chemistry,' of Osaka University.
1 Scherf, U. \& Neher, D. (eds.) Polyfluorenes (Springer: Berlin, Heiderberg, 2008).

2 Grimsdale, A. C. \& Müllen, K. Polyphenylene-type emissive materials: poly(paraphenylene)s, polyfluorenes, and ladder polymers. Adv. Polym. Sci. 199, 1 (2006).

3 Oda, M., Nothofer, H.- G., Lieser, G., Scherf, U., Meskers, S. C. J. \& Neher, D. Circularly polarized electroluminescence from liquid-crystalline chiral polyfluorenes. Adv. Mater. 12, 362-365 (2000)

4 Oda, M., Meskers, S. C. J., Nothofer, H. G., Scherf, U. \& Neher, D. Chiroptical properties of chiral-substituted polyfluorenes. Synth. Met 111-112, 575-577 (2000).

5 Tang, H.- Z., Fujiki, M. \& Sato, T. Thermodriven conformational transition of optically active poly[2,7-|\{9,9-bis[(S)-2-methyloctyl]\}\}fluorene] in solution. Macromolecules 35, 6439-6445 (2002).

6 Wu, L., Sato, T., Tang, H.- Z. \& Fujiki, M. Conformation of a polyfluorene derivative in solution. Macromolecules 37, 6183-6188 (2004).

7 Wu, L. \& Sato, T. Aggregation-induced circular dicroism of optically active polyfluorene derivatives in solution. Kobunshi Ronbunshu 63, 505-511 (2006).

8 Lieser, G., Oda, M., Miteva, T., Meisel, A., Nothofer, H.- G., Scherf, U. \& Neher, D. Ordering, graphoepitaxial orientation, and conformation of a polyfluorene derivative of the 'hairy-rod' type on an oriented substrate of polyimide. Macromolecules 33, 4490-4495 (2000).

9 Hong, S. Y., Kim, D. Y., Kim, C. Y. \& Hoffmann, R. Origin of the broken conjugation in m-phenylene linked conjugated polymers. Macromolecules 34, 6474-6481 (2001).

10 Knaapila, M. \& Winokur, M. J. Structure and morphology of polyfluorenes in solutions and the solid state. Adv. Polym. Sci. 212, 227-272 (2008).

11 Lakhwani, G., Meskers, S. C. J. \& Janssen, R. A. J. Circular differential scattering of light in films of chiral polyfluorene. J. Phys. Chem. B 111, 5124-5131 (2007).

12 Langeveld-Voss, B. M. W., Waterval, R. J. M., Janssen, R. A. J. \& Meijer, E. W. Principles of 'majority rules' and 'sergeants and soldiers' applied to the aggregation of optically active polythiophenes: evidence for a multichain phenomenon. Macromolecules 32, 227-230 (1999).

13 Nakashima, H., Fujiki, M., Koe, J. R. \& Motonaga, M. Solvent and temperature effects on the chiral aggregation of poly(alkylarylsilane)s bearing remote chiral groups. J. Am. Chem. Soc. 123, 1963-1969 (2001).

14 Goto, H. \& Yashima, Y. Electron-induced switching of the supramolecular chirality of optically active polythiophene aggregates. J. Am. Chem. Soc. 124, 7943-7949 (2002).

15 Peng, W., Motonaga, M. \& Koe, J. R. Chirality control in optically active polysilane aggregates. J. Am. Chem. Soc. 126, 13822-13826 (2004).

16 Yamakawa, H. Modern Theory of Polymer Solutions Rice S.A. (ed). (Harper \& Row: New York, 1971).

17 Strazielle, C. in Light Scattering from Polymer Solutions Huglin M.B. (ed). (Academic Press: London \& New York, 1972).

18 Flory, P. J. Principles of Polymer Chemistry (Cornell University Press: Ithaca, New York, 1953).

19 Dias, F. B., Morgado, J., Maçanita, A. L., da Costa, F. P., Burrows, H. D. \& Monkman, A. P. Kinetics and thermodynamics of poly $(9,9$-dioctylfluorene) $\beta$-phase formation in dilute solution. Macromolecules 39, 5854-5864 (2006).

20 Sato, T., Terao, K., Teramoto, A. \& Fujiki, M. Molecular properties of helical polysilylenes in solution. Polymer 44, 5477-5495 (2003).

21 Wesslau, H. Die molekulargewichtsverteilung einiger niederdruckpolyäthylene. Makromol. Chem. 20, 111-120 (1956).

22 Brandrup, J. \& Immergut, E. H. (eds). Polymer Handbook 3rd edn. (John Wiley \& Sons: New York, 1989).

23 Prod, G. In: Small Angle X-ray Scattering Glatter O., Kratky O.(eds). (Academic Press: London, 1982).

24 Debye, P. Light scattering in soap solutions. Ann. N.Y. Acad. Sci. 51, 575-592 (1949).

25 McLachlan, A. D. Retarded dispersion forces between molecules. Proc. Roy. Soc. London A271, 387-401 (1963).

26 McLachlan, A. D. Retarded dispersion forces in dielectrics at finite temperatures. Proc. Roy. Soc. London A274, 80-90 (1963).

27 Imura, H. \& Okano, K. van der Waals-Lifshitz forces between anisotropic ellipsoidal particles. J. Chem. Phys. 58, 2763-2776 (1973).

28 Israelachvili, J. N. Intermolecular and Surface Forces 2nd edn. (Academic Press: London, 1992).

29 Osipov, M. A. Theory for cholesteric ordering in lyotropic liquid crystals. II. Nuovo Cimento D 10, 1249-1262 (1988).

30 Osipov, M. A. Molecular theory of solvent effect on cholesteric ordering in lyotropic polypeptide liquid crystals. Chem. Phys. 96, 259-270 (1985).

31 Osipov, M. A. in Liquid Crystalline and Mesomorphic Polymers Shibaev V.P., Lam L. (eds). (Springer: Berlin \& Heidelberg, 1994), Chapter 1.

32 Lifson, S., Andreola, C., Peterson, N. C. \& Green, M. M. Macromolecular stereochemistry: helical sense preference in optically active polyisocyanates. Amplification of a conformational equilibrium deuterium isotope effect. J. Am. Chem. Soc. 111, 8850-8858 (1989).

33 Morino, K., Maeda, K., Okamoto, Y., Yashima, E. \& Sato, T. Temperature dependence of helical structures of poly(phenylacetylene) derivatives bearing an optically active substituent. Chem. Eur. J. 8, 5112-5120 (2002). 